\title{
MicroRNAs as outcome predictors in patients with metastatic colorectal cancer treated with bevacizumab in combination with FOLFOX
}

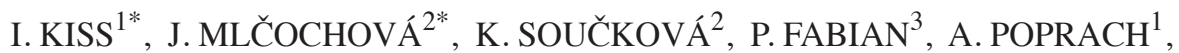 \\ J. HALAMKOVA ${ }^{1}$, M. SVOBODA ${ }^{1}$, R. VYZULA ${ }^{1}$ and O. SLABY ${ }^{1,2}$ \\ ${ }^{1}$ Department of Comprehensive Cancer Care, Masaryk Memorial Cancer Institute, 65653 Brno; \\ ${ }^{2}$ Department of Molecular Medicine, Central European Institute of Technology, Masaryk University, 62500 Brno; \\ ${ }^{3}$ Department of Clinical and Experimental Pahology, Masaryk Memorial Cancer Institute, 65653 Brno, Czech Republic
}

Received January 4, 2017; Accepted March 30, 2017

DOI: $10.3892 / 01.2017 .6255$

\begin{abstract}
Bevacizumab is a humanized anti-vascular endothelial growth factor monoclonal antibody, used in combination with a oxaliplatin-based chemotherapy in the treatment of metastatic colorectal cancer (mCRC). The aim of the present study was to identify microRNA (miRNA)-based predictive biomarkers of therapy response in order to avoid unnecessary and costly therapy to non-responding patients. High-throughput miRNA microarray profiling (Affymetrix miRNA array) was performed on a discovery cohort of patients with mCRC. The discovery cohort was $(n=20)$ divided into either responding $(\mathrm{n}=10)$ or non-responding $(\mathrm{n}=10)$ groups of bevacizumab/5-flourouracil, leucovorin, oxaliplatin (FOLFOX) treatment according to Response Evaluation Criteria in Solid Tumors criteria. Validation of candidate miRNAs was performed on an independent cohort of 41 patients with $\mathrm{mCRC}$ using quantitative reverse transcription polymerase chain reaction. Normalized data were subjected to receiver operating characteristic and Kaplan-Meier analyses. In total, 67 miRNAs were identified to be differentially expressed when miRNA expression was compared between responding and non-responding patients to bevacizumab/FOLFOX treatment $(\mathrm{P}<0.05)$. A total of 7 miRNAs were chosen for independent validation, which confirmed significantly higher expression of miR-92b-3p, miR-3156-5p, miR-10a-5p and miR-125a-5p $(\mathrm{P}<0.005)$ in tumor tissue of responding patients
\end{abstract}

Correspondence to: Dr Ondrej Slaby, Department of Molecular Medicine, Central European Institute of Technology, Masaryk University, University Campus Bohunice, Kamenice 5, 62500 Brno, Czech Republic

E-mail: on.slaby@gmail.com

${ }^{*}$ Contributed equally

Key words: bevacizumab, metastatic colorectal cancer, microRNA, progression-free survival, predictive biomarker compared with non-reponding patients. Using the combination of miRNAs, the present study identified responders to the therapy with sensitivity $82 \%$ and specificity $64 \%$ (area under the curve $=0.8015$ ). In conclusion, 4 predictive miRNAs associated with progression-free survival (PFS) were identified in patients with mCRC treated with bevacizumab/FOLFOX. Following further independent validations, detection of these miRNA may enable identification of patients with mCRC who may potentially benefit from the therapy.

\section{Introduction}

Colorectal cancer (CRC) is one of the most commonly diagnosed types of cancer worldwide (1). Despite increases in early diagnosis due to implementation of screening programmes, $>5 \%$ of patients present with distant and in the majority of cases unresectable, metastatic lesions, representing a common cause of cancer-associated mortality. Patients with mCRC, who are not able to undergo radical resection of metastases, are subjected to standard system chemotherapy with or without a targeted agent (2). Bevacizumab is a recombinant humanized monoclonal antibody (moAb) that blocks angiogenesis by inhibiting vascular endothelial growth factor (VEGF) or VEGF-A. When used in combination with standard chemotherapy (fluorouracil/capecitabine and oxaliplatin), bevacizumab treatment resulted in a longer progression free survival (PFS) of patients with mCRC compared with treatment with chemotherapy alone (3). However, the benefit of the treatment is modest and causes toxicity in patients when used unselectively. Additionally, almost all treated patients will develop secondary resistance to bevacizumab (3-5). Other therapeutic alternatives, including moAb against the epidermal growth factor receptor (EGFR) cetuximab, a fully humanised EGFR-antibody panitumumab or other targeted agents $(6,7)$ may be used for treatment if there is a possibility of distinguishing between patients who may or may not benefit from bevacizumab/5-flourouracil, leucovorin, oxaliplatin (FOLFOX) regimen.

MicroRNAs (miRNAs) are small, non-coding RNAs that regulate gene expression post-transcriptionally. Numerous 
miRNAs function as either oncogenes or tumor suppressors (8). miRNAs serve an important role in the regulation of the majority of cellular processes. Variability in miRNA expression has been demonstrated to affect various cancer types including CRC (9). miRNAs are relatively resistant to decay and so may be detected and quantified in tissues and body fluids (10). This property makes miRNAs candidate biomarkers with diagnostic, prognostic and predictive potential, and also makes them promising therapeutic targets (11). To the best of our knowledge, there have been two previous studies published to date that focused on the ability of miRNAs to predict responses to bevacizumab-based therapy in metastatic CRC $(12,13)$.

The aim of the present study was to identify tissue miRNAs associated with PFS in patients with mCRC treated specifically with a bevacizumab/FOLFOX regimen in order to establish an effective tool for distinguishing patients who would benefit from this first-line treatment for mCRC.

\section{Materials and methods}

Patients, tissue samples and study design. A total of 61 Caucasian patients with $\mathrm{mCRC}$ treated with combined therapy bevacizumab/FOLFOX in a standard regimen as previously described (3) were enrolled in the study. The patients included 40 males and 21 females; the mean \pm standard deviation age was $63 \pm 8$ years with a range of 34-74 years. All patients were treated for generalized-radically inoperable metastatic colorectal cancer at the Masaryk Memorial Cancer Institute (Brno, Czech Republic) between January 2006 and December 2012. All patients had a well-documented history of treatment, including the effect of treatment and toxicity. A total of 63 tissue samples obtained from the patients during surgical resection of the primary tumor were used in the present study. The clinicopathological characteristics of patients are summarized in Table I. Response to the bevacizumab/FOLFOX treatment was assessed according to Response Evaluation Criteria in Solid Tumors (RECIST) criteria and duration of PFS (14). The patients were divided into two groups: i) Complete or partial response (responders), and ii) stable disease and progressive disease (non-responders). The tissue samples were fixed in $10 \%$ formalin immediately following extraction. The discovery $(n=20$, including 10 responding and 10 non-responding patients) and validation cohorts $(n=41)$ of patients with $\mathrm{mCRC}$ were set up retrospectively based on the RECIST response and PFS. The study protocol was approved by Masaryk Memorial Cancer Institute Ethics Committee, and written informed consent was obtained from all patients enrolled in the study.

RNA isolation. The total RNA enriched for small RNA fraction was isolated from formalin-fixed paraffin embedded (FFPE) tissue samples of primary CRC tumors, which were surgically resected as a first treatment modality. The first step of the protocol was xylene deparaffinization, followed by purification by mirVana ${ }^{\mathrm{TM}}$ miRNA Isolation kit (Ambion; Thermo Fisher Scientific, Inc., Waltham, MA, USA) according to the manufacturer's protocol. The concentration and purity of RNA was determined by UV spectrophotometry using an ND-1000 machine (Nanodrop; Thermo Fisher Scientific, Inc., Wilmington, DE, USA). RNA samples with sufficient purity
Table I. Patient characteristics.

\begin{tabular}{|c|c|c|c|}
\hline Variable & $\begin{array}{c}\text { All } \\
\text { patients }\end{array}$ & $\begin{array}{c}\text { Discovery } \\
\text { cohort }\end{array}$ & $\begin{array}{c}\text { Validation } \\
\text { cohort }\end{array}$ \\
\hline Total, $\mathrm{n}$ & 61 & 20 & 41 \\
\hline \multicolumn{4}{|l|}{ Gender, $\mathrm{n}$} \\
\hline Male & 40 & 12 & 28 \\
\hline Female & 21 & 8 & 13 \\
\hline \multicolumn{4}{|l|}{ Age, years } \\
\hline $\begin{array}{l}\text { Mean } \pm \text { standard } \\
\text { deviation }\end{array}$ & $63 \pm 8$ & $60 \pm 10$ & $64 \pm 6$ \\
\hline Range & $34-74$ & $34-74$ & $51-74$ \\
\hline \multicolumn{4}{|l|}{ KRAS, n } \\
\hline Wild-type & 33 & 9 & 24 \\
\hline Mutated & 19 & 6 & 13 \\
\hline Not available & 9 & 5 & 4 \\
\hline \multicolumn{4}{|l|}{ RECIST response, $\mathrm{n}$} \\
\hline $\mathrm{CR} / \mathrm{PR}$ & 34 & 10 & 24 \\
\hline $\mathrm{SD} / \mathrm{PD}$ & 27 & 10 & 17 \\
\hline \multicolumn{4}{|l|}{$\begin{array}{l}\text { PFS, median } \\
\text { (interquartile } \\
\text { range) }\end{array}$} \\
\hline Responders $^{\mathrm{a}}$ & $25(20-44)$ & $45(33-55)$ & $20(19-25)$ \\
\hline Non-responders ${ }^{\mathrm{b}}$ & $6(3-8)$ & $3(2-6)$ & $7(4-8)$ \\
\hline
\end{tabular}

${ }^{\text {aPFS }} \geq 12$ months; ${ }^{\text {bPFS }}<12$ months. RECIST, Response Evaluation Criteria in Solid Tumors; CR, complete response; PR, partial response $\mathrm{SD}$, stable disease; $\mathrm{PD}$, progressive disease; PFS, progression-free survival.

(260/280 ratio $>2.0$ and $260 / 230$ ratio $>1.8$ ) were used for further study.

miRNA microarray profiling. miRNA microarray profiling was conducted using Affymetrix GeneChip miRNA 3.0 arrays (Affymetrix, Inc., Santa Clara, CA, USA) containing 5,607 probe sets for human pre-miRNA, miRNAs, small nuclear RNA, and small Cajal body-specific RNA. These Affymetrix miRNA arrays provide $100 \%$ coverage of the miRNAs in the miRBase (version 17; http://www.mirbase. org). Probe sets that were deleted in a more recent version of miRBase (version 21) were excluded from the analysis. All steps of the procedure were performed according to the Affymetrix standardized protocol for miRNA 3.0 arrays.

RT-qPCR quantification. Commercial TaqMan Advanced miRNA assays for miR-877-5p, miR-642a-3p, miR-642b-3p, miR-92b-3p, miR-3156-5p, miR-10a-5p and miR-125a-5p, based on stem-loop reverse transcription, and TaqMan Universal PCR Master mix (all from Applied Biosystems; Thermo Fisher Scientific, Inc., Waltham, MA, USA) were used for the individual quantification of the miRNAs selected in the discovery phase of the study. RT-qPCR was performed with QuantStudio 12 K Flex Real-Time PCR system (Applied Biosystems; Thermo Fisher Scientific, Inc.) using the temperature profiles 


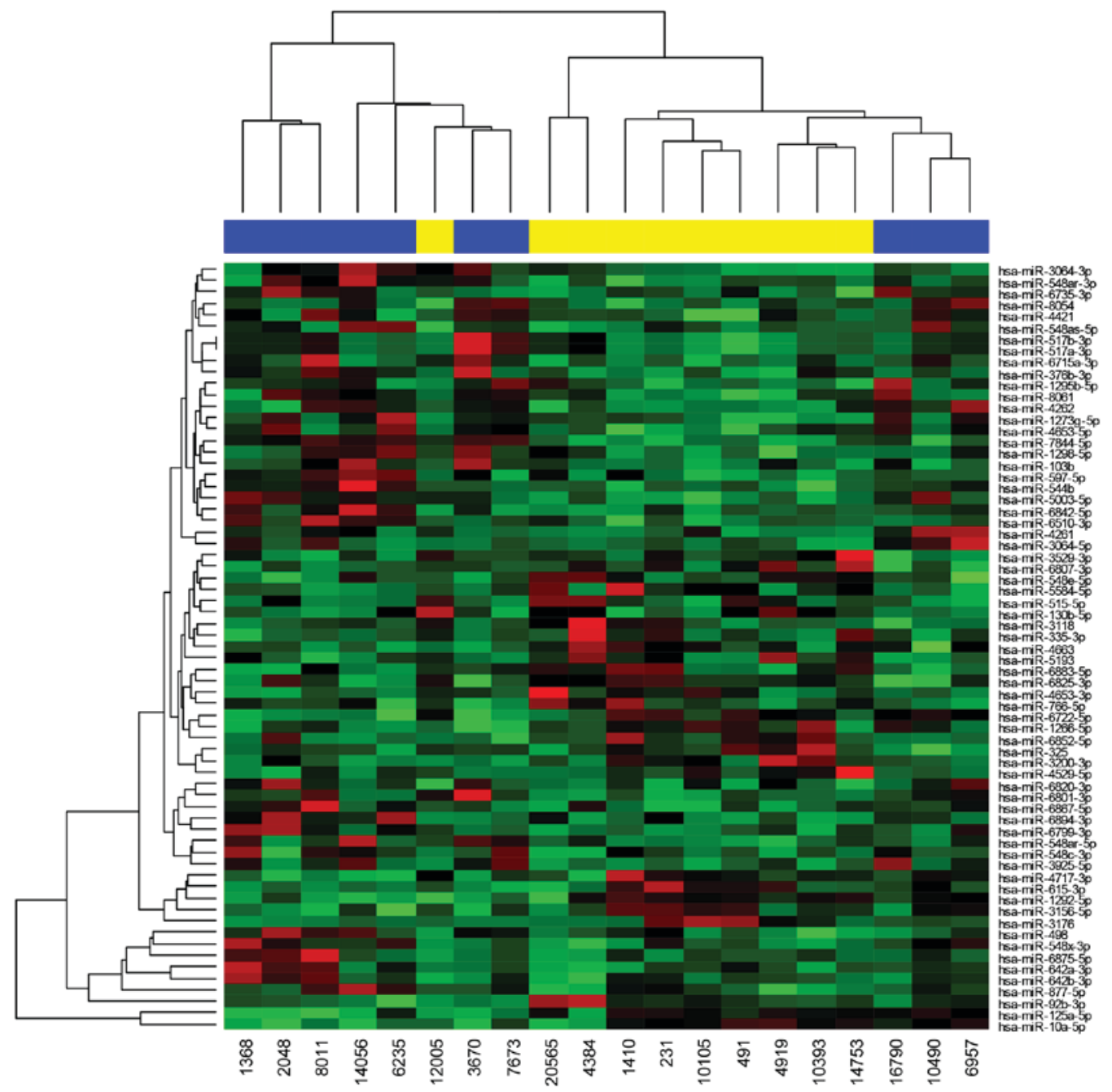

Figure 1. Hierarchical clustering analysis and heat map based on the expression profiles of 67 miRNAs in responding and non-responding patients to bevacizumab/5-flourouracil, leucovorin, oxaliplatin treatment. Cluster analysis grouped samples and miRNAs according to similarity in expression. miRNAs are in rows and samples in columns. The miRNA clustering tree is indicated on the left, and the sample clustering tree is at the top. Red color represents upregulated expression, and green marks downregulated genes. Yellow indicates responders, and blue indicates non-responders. miRNA, microRNA.

from the manufacturer's protocols. PCR was run in triplicate. Average threshold cycles and standard deviation values were calculated as described in the subsequent section.

Normalization and statistical analysis. The Affymetrix raw data were normalized using the robust multichip average algorithm from 'oligo' Bioconductor package (15) in $\mathrm{R}$ version 3.0.1 (16). Normalized miRNA expression data were evaluated using Bioconductor LIMMA differential expression analysis (17). $\mathrm{P}<0.05$ was selected according to the potential of identified miRNAs to accurately discriminate responding and non-responding patients in subsequent hierarchical clustering analysis (15). In the validation phase of the study, mean expression levels of candidate miRNAs in RT-qPCR quantification were normalized to the expression of miR-6098 as the endogenous control as per a previously described method (18). miR-6098 was selected by the use of GeneNorm and NormFinder algorithms to be uniformly expressed in all samples in the discovery cohort (19). Normalized expression data were evaluated by the Mann-Whitney U test, by a bidirectional stepwise logistic regression model to establish the combined miRNA diagnostic score (4-miRNA-based diagnostic score $=1.9086-0.0037 \mathrm{x}$ miR-92a-3p $-1.1517 \mathrm{x}$ miR-3156-5p-0.0892x miR-10a-5p-0.0986x miR-125a-5p) and by receiver operating characteristic (ROC) analysis to identify cut-off values and subsequently by Kaplan-Meier analysis followed by log-rank test for survival analysis (GraphPad Prism version 5.0; GraphPad Software, Inc., La Jolla, CA, USA). $\mathrm{P}<0.05$ was considered to indicate a statistically significant difference.

\section{Results}

miRNAs are differentially expressed between responders and non-responders to FOLFOX-bevacizumab therapy. High-throughput miRNA microarray profiling was performed on a discovery cohort of 20 patients. These individuals were treated with a bevacizumab/FOLFOX regimen and were divided into responding or non-responding groups. LIMMA differential expression analysis of normalized expression data identified 67 miRNAs that were differentially expressed in responding and non-responding patients (Fig. 1; Table II). A total of 7 candidate miRNAs (miR-887-5p, miR-642a-3p, miR-642b-3p, miR-92b-3p, miR-3156-5p, miR-10a-5p and 
Table II. miRs identified to be differentially expressed in patients responding and non-responding to bevacizumab/FOLFOX treatment.

\begin{tabular}{|c|c|c|}
\hline $\operatorname{miR}$ & Fold-change & P-value \\
\hline hsa-miR-5003-5p & 1.48 & 0.001 \\
\hline hsa-miR-7844-5p & 1.49 & 0.002 \\
\hline hsa-miR-548x-3p & 2.68 & 0.003 \\
\hline hsa-miR-3925-5p & 1.99 & 0.003 \\
\hline hsa-miR-6825-3p & 0.66 & 0.005 \\
\hline hsa-miR-1273g-5p & 1.36 & 0.006 \\
\hline hsa-miR-4262 & 1.37 & 0.007 \\
\hline hsa-miR-498 & 3.20 & 0.008 \\
\hline hsa-miR-4653-5p & 1.34 & 0.009 \\
\hline hsa-miR-6842-5p & 1.45 & 0.01 \\
\hline hsa-miR-6807-3p & 0.71 & 0.01 \\
\hline hsa-miR-544b & 1.31 & 0.012 \\
\hline hsa-miR-877-5p ${ }^{a}$ & 2.85 & 0.013 \\
\hline hsa-miR-6820-3p & 1.46 & 0.014 \\
\hline hsa-miR-548as-5p & 1.36 & 0.014 \\
\hline hsa-miR-5193 & 0.62 & 0.014 \\
\hline hsa-miR-3529-3p & 0.72 & 0.015 \\
\hline hsa-miR-515-5p & 0.70 & 0.017 \\
\hline hsa-miR-642a-3pa & 5.53 & 0.017 \\
\hline hsa-miR-8054 & 1.32 & 0.017 \\
\hline hsa-miR-3176 & 0.45 & 0.017 \\
\hline hsa-miR-3064-5p & 1.50 & 0.018 \\
\hline hsa-miR-548e-5p & 0.74 & 0.019 \\
\hline hsa-miR-4653-3p & 0.65 & 0.019 \\
\hline hsa-miR-6722-5p & 0.66 & 0.02 \\
\hline hsa-miR-6883-5p & 0.66 & 0.023 \\
\hline hsa-miR-517a-3p & 1.37 & 0.024 \\
\hline hsa-miR-517b-3p & 1.37 & 0.024 \\
\hline hsa-miR-548c-3p & 1.49 & 0.024 \\
\hline hsa-miR-548ar-5p & 1.51 & 0.024 \\
\hline hsa-miR-8061 & 1.29 & 0.024 \\
\hline hsa-miR-325 & 0.77 & 0.028 \\
\hline hsa-miR-4663 & 0.72 & 0.028 \\
\hline hsa-miR-6894-3p & 1.70 & 0.028 \\
\hline hsa-miR-92b-3p ${ }^{a}$ & 0.32 & 0.029 \\
\hline hsa-miR-3156-5 $\mathrm{p}^{\mathrm{a}}$ & 0.52 & 0.029 \\
\hline hsa-miR-615-3p & 0.57 & 0.029 \\
\hline hsa-miR-3118 & 0.68 & 0.029 \\
\hline hsa-miR-6510-3p & 1.35 & 0.03 \\
\hline hsa-miR-4717-3p & 0.59 & 0.03 \\
\hline hsa-miR-6852-5p & 0.66 & 0.03 \\
\hline hsa-miR-548ar-3p & 1.39 & 0.031 \\
\hline hsa-miR-597-5p & 1.32 & 0.032 \\
\hline hsa-miR-4261 & 1.63 & 0.033 \\
\hline hsa-miR-6799-3p & 1.49 & 0.034 \\
\hline hsa-miR-1298-5p & 1.30 & 0.034 \\
\hline hsa-miR-6875-5p & 3.18 & 0.034 \\
\hline hsa-miR-130b-5p & 0.74 & 0.036 \\
\hline hsa-miR-6867-5p & 1.69 & 0.036 \\
\hline hsa-miR-5584-5p & 0.81 & 0.037 \\
\hline
\end{tabular}

Table II. Continued.

\begin{tabular}{lcr}
\hline miR & Fold-change & P-value \\
\hline hsa-miR-642b-3p & 3.49 & 0.037 \\
hsa-miR-3064-3p & 1.30 & 0.038 \\
hsa-miR-4529-5p & 0.72 & 0.038 \\
hsa-miR-103b & 1.38 & 0.038 \\
hsa-miR-376b-3p & 1.33 & 0.038 \\
hsa-miR-766-5p & 0.67 & 0.039 \\
hsa-miR-3200-3p & 0.75 & 0.04 \\
hsa-miR-6735-3p & 1.27 & 0.04 \\
hsa-miR-6801-3p & 1.38 & 0.04 \\
hsa-miR-10a-5p & 0.20 & 0.044 \\
hsa-miR-1266-5p & 0.75 & 0.045 \\
hsa-miR-1295b-5p & 1.27 & 0.047 \\
hsa-miR-335-3p & 0.68 & 0.047 \\
hsa-miR-6715a-3p & 1.34 & 0.047 \\
hsa-miR-4421 & 1.22 & 0.049 \\
hsa-miR-125a-5p & 0.19 & 0.049 \\
hsa-miR-1292-5p & 0.62 & 0.05 \\
\hline
\end{tabular}

${ }^{a}$ miRNAs selected for independent validation. miR, microRNA.

miR-125a-5p) were chosen for further independent validation by RT-qPCR according to the following criteria: Fold change $>2$, $\mathrm{P}<0.05$ and Affymetrix miRNA array signal intensity $>3$.

Association of miRNAs with response to bevacizumab/FOLFOX therapy. The expression levels of 7 candidate miRNAs identified in the discovery phase of the present study were determined in the validation cohort of 41 patients by RT-qPCR ( $<<0.01 ; \mathrm{Cq}<35)$. A total of 4 miRNAs (miR-92b-3p, miR-3156-5p, miR-10a-5p, and miR-125a-5p) were confirmed to be associated with radiological response according to RECIST criteria ( $\mathrm{P}<0.005$; Fig. 2A-D; Table III). All 4 miRNAs were upregulated in responders to bevacizumab/FOLFOX therapy. The association with RECIST response of the remaining 3 miRNAs included in the validation phase did not reach statistical significance: miR-877-5p ( $\mathrm{P}=0.4430)$, miR-642a-3p $(\mathrm{P}=0.1974)$ and miR-642b-3p $(\mathrm{P}=0.3688)$. Kaplan-Meier analysis demonstrated that higher levels of miR-125a-5p, miR-10a-5p, miR-3156-5p and miR-92b-3p were also significantly associated with extended PFS (Fig. 2E-H; Table III).

Subsequent ROC analysis revealed that the usage of 4-miRNA-combined diagnostic score, as established by a bidirectional stepwise logistic regression, enabled the identification of responders to bevacizumab/FOLFOX therapy with a sensitivity of $82 \%$ and specificity of $64 \%$ [area under the curve $(\mathrm{AUC})=0.8015 ; \mathrm{P}=0.008$, cut-off value $=-0.466 ;$ Fig. 3$]$. Based on this diagnostic score, the median PFS in responders was 20 months vs. 7 months for non-responders.

\section{Discussion}

The aim of the present study was to identify candidate miRNAs that would assist in identifying patients with $\mathrm{mCRC}$ who 
A
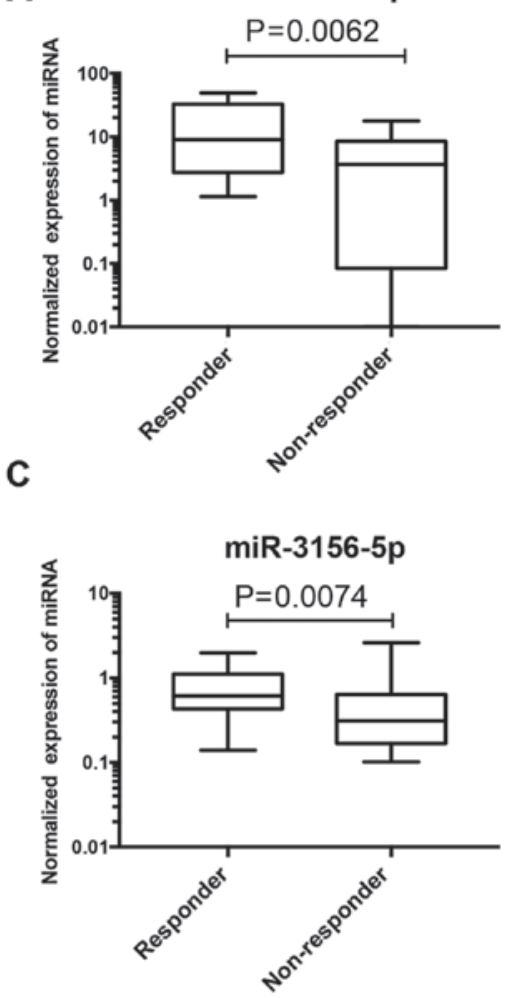

E

miR-125a-5p

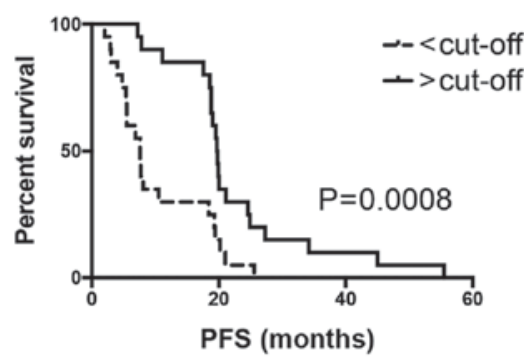

G

miR-3156-5p

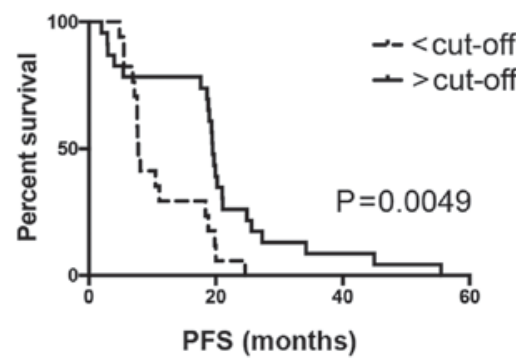

B

miR-10a-5p
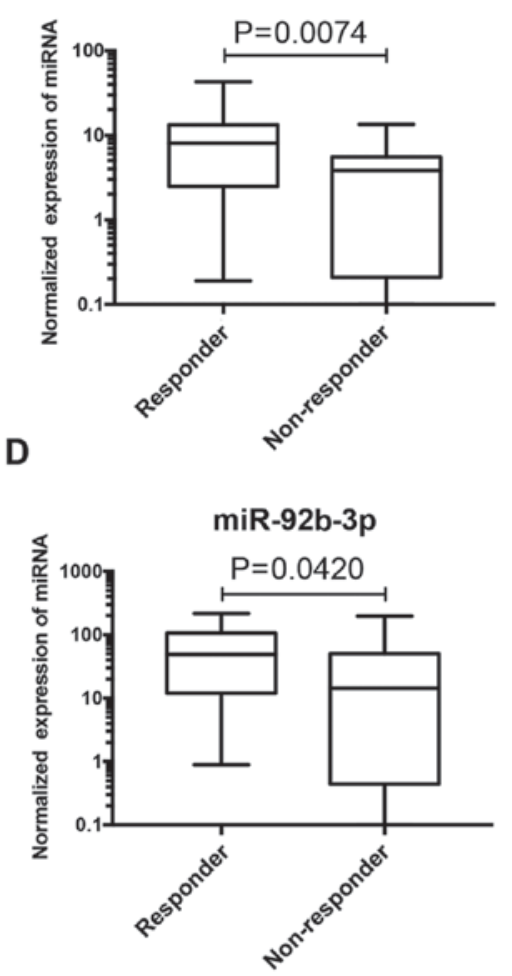

F

miR-10a-5p

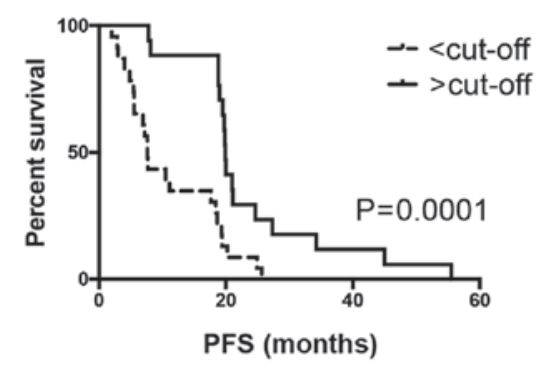

H

miR-92b-3p

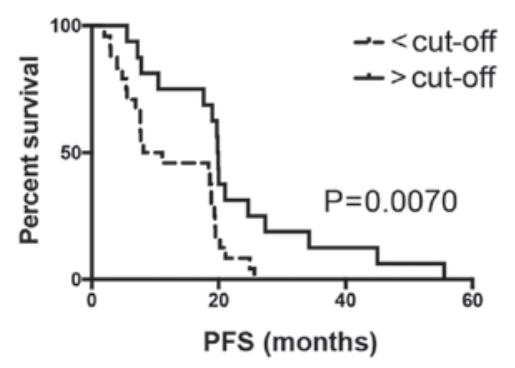

Figure 2. miRNAs associated with therapy response. Normalized expression of (A) miR-125a-5p, (B) miR-10a-5p, (C) miR-3156-5p, and (D) miR-92b-3p in patients responding and non-responding to bevacizumab/FOLFOX therapy. Quantification of quantitative reverse transcription polymerase chain reaction was normalized to miR-6098 expresion. Kaplan-Meier survival curves estimating time to progression in bevacizumab/FOLFOX-treated patients with metastatic colorectal cancer $(n=41)$ according to $(\mathrm{E})$ miR-125a-5p, $(\mathrm{F})$ miR-10a-5p, $(\mathrm{G})$ miR-3156-5p, and (H) miR-92b-3p tumor tissue expression levels. miRNA, microRNA; FOLFOX, 5-flourouracil, leucovorin, oxaliplatin; PFS, progression-free survival.

were likely to respond to targeted antiangiogenic treatment in combination with a FOLFOX regimen. Oxaliplatin-based chemotherapy combined with bevacizumab is a common choice for patients with mCRC. Unfortunately, the benefit for patients is rather limited as at present, it has not been possible to identify patients most likely to benefit from this regimen (3). The results of the present indicate that specific miRNAs exhibit a predictive value in this setting. 
Table III. Validation of miRNAs identified in the discovery phase $(n=41)$ of the study.

\begin{tabular}{|c|c|c|c|c|c|}
\hline Parameters & Number of patients & Median PFS (months) & Log-rank P-value & Hazard ratio & $95 \% \mathrm{CI}$ \\
\hline \multicolumn{6}{|l|}{$\operatorname{miR}-125 a-5 p$} \\
\hline Low, $<8.7$ & 20 & 7.6 & 0.0008 & 2.652 & $1.323-5.313$ \\
\hline High, $\geq 8.7$ & 21 & 19.8 & & & \\
\hline \multicolumn{6}{|l|}{ miR-10a-5p } \\
\hline Low, $<6.2$ & 17 & 7.6 & 0.0001 & 2.805 & $1.447-5.434$ \\
\hline High, $\geq 6.2$ & 24 & 20.0 & & & \\
\hline \multicolumn{6}{|l|}{ miR-3156-5p } \\
\hline Low, $<0.4$ & 24 & 7.8 & 0.0049 & 2.259 & $1.101-4.636$ \\
\hline High, $\geq 0.4$ & 17 & 19.5 & & & \\
\hline \multicolumn{6}{|l|}{ miR-92b-3p } \\
\hline Low, $<0.4$ & 17 & 9.6 & 0.0070 & 2.215 & $1.178-4.166$ \\
\hline High, $\geq 0.4$ & 24 & 19.9 & & & \\
\hline
\end{tabular}

PFS, progression-free survival; CI, confidence interval; miRNA, microRNA.

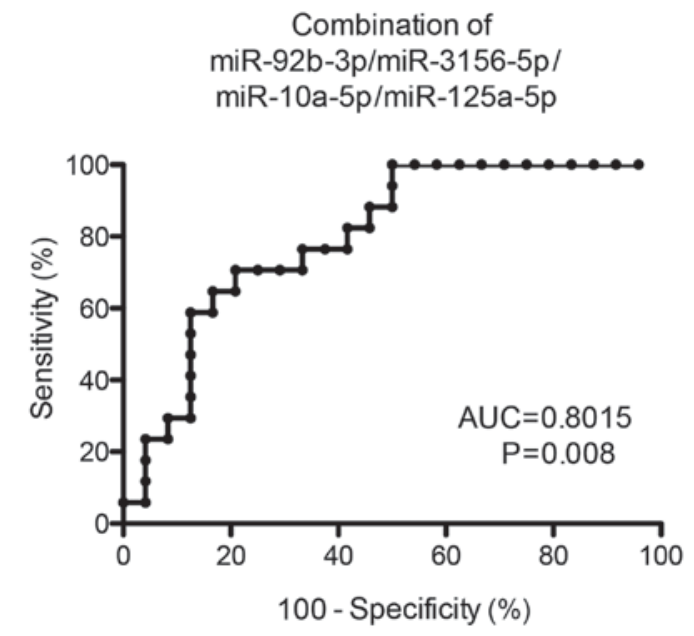

Figure 3. Receiver operating characteric analysis of the use of miR-92b-3p, miR-3156-5p, miR-10a-5p and miR-125a-5p combination in discriminating between responders and non-responders to bevacizumab/5-flourouracil treatment. AUC, area under the curve.

By global miRNA microarray profiling of FFPE tumor tissues and comparing miRNA expression between responders and non-responders to bevacizumab/FOLFOX therapy, a total of 67 miRNAs with significantly different expression were identified. However, only 7 miRNAs fulfilled the criteria for selection of candidate miRNAs for validation. These 7 miRNA were further investigated in the validation phase of the present study. The independent validation of these miRNAs in the cohort of 41 patients with mCRC confirmed the ability to distinguish patients with different responses to bevacizumab/FOLFOX treatment only in the case of 4 miRNAs (miR-125a-5p, miR-10a-5p, miR-3156-5p and miR-92b-3p). In the group of responders, significantly higher levels of these 4 miRNAs was observed compared with non-responders. The analytical performance of the individual miRNAs was unsatisfactory (AUC <0.75). However when analyzed in combination, these 4 miRNAs enabled the sensitive and specific identification of patients with $\mathrm{mCRC}$ responsive to bevacizumab/FOLFOX treatment $(\mathrm{AUC}=0.8015$; sensitivity, 82\%; specificity, 64\%). Notably, Boisen et al (12) did not identify any of the predictive miRNAs of the present study, and observed only miR-664-3p and miR-455-5p to be associated with overall survival in patients with mCRC treated with capecitabine, oxaliplatin and bevacizumab.

As the course of the survival curves appears similar for all significant miRNAs identified by the present study, it is hypothesized that the miRNAs are associated with the same biological feature, for example high angiogenic density, responsible for sensitivity to anti-angiogenic treatment. In addition, these miRNAs may share identical mechanism of secondary resistance, represented by the significant increase in progression rate following 20 months of therapy.

Association between miR-10a-5p expression and clinical parameters in CRC has been described previously (20). The high expression of miR-10b-5p has been associated with tumors located in the right colon relative to the left colon and rectum, but no association to metastasis-free survival has been observed (20). In agreement with the observations of the present study, the expression levels of miR-10a-5p in tumor FFPE samples were demonstrated to be $>2$-fold higher in progession-free patients compared with patients with progressive disease in bladder cancers (21). The prognostic significance of miR-10a-5p was also confirmed in acute myeloid leukemia, where the serum expression levels of this miRNA were significantly higher in comparison with a control group (22). In patients with recurrent platinum-resistant ovarian cancer, higher expression levels of miR-10a-5p were noted in peripheral blood plasma and associated with a sensitivity to decitabine followed by carboplatin chemotherapy (23).

Changes in miR-125a-5p expression levels have been identified in several types of cancer. Gattolliat et al (24) and Ozcan et al (25) suggested that miR-125a-5p was deregulated in CRC tissue samples. In addition, downregulated miR-125a-5p was significantly associated with gastric cancer metastases (26) and was significantly downregulated in the 
serum of patients with lung cancer $(\mathrm{P}<0.001)(27)$, indicating its potential tumor-suppressive role in cancer. In the present study, a higher level of miR-125a-5p were observed to be associated with an improved outcome in patients with $\mathrm{mCRC}$ treated with bevacizumab/FOLFOX. Understanding the processes accompanying hypoxia is one of the main goals of studies investigating cancer, as it is known that hypoxia serve a role in the resistance to chemotherapy and radiotherapy (28). Using the grade IV glioma U87MG cell line, it was demonstrated that miR-92b-3p was downregulated by $>2$-fold under hypoxic conditions (29), suggesting miR-92-3p expression to be indicative of hypoxic environment. At present, miR-3156-5p expression has not been described in association with cancer.

There are certain potential limitations of the present study, and a number of issues need to be addressed in order to establish miRNAs as clinical diagnostic and predictive tools. The validation cohort in the present study was not large enough to support the clinical utility of the 4-miRNA predictive panel identified. Additionally, all samples were enrolled from a single center, and all patients were of the same ethnicity (European descent). There is a need for a prospective, multi-centric study involving large independent cohorts. As the levels of circulating miR-126 have been observed to be associated with the response to bevacizumab treatment (14), it would be also of interest to evaluate the predictive value of the tissue miRNAs identified in the predictive panel from the present study in peripheral blood or blood serum/plasma samples, as a potentially non-invasive diagnostic option.

In conclusion, 4 miRNAs (miR-92b-3p, miR-3156-5p, miR-10a-5p and miR-125a-5p) were identified to exhibit higher expression levels in the tumors of patients who responded to bevacizumab/FOLFOX therapy compared with non-responders. Subsequent to further independent validations, detection of these miRNA would enable the identification of patients with mCRC who may potentially benefit from this therapy. These miRNAs may also serve as novel candidates for targeted therapeutic interventions in patients with mCRC, even though the role of these miRNAs and the signaling pathways these miRNAs regulate remain to be clarified.

\section{Acknowledgements}

The present study was financially supported by the Czech Ministry of Health (grant no. 16-31765A), project MZ CR-RVO (grant no. MOU, 00209805) and the Ministry of Education, Youth and Sports of the Czech Republic under the project Central European Institute of Technology 2020 (grant no. LQ1601).

\section{References}

1. Ferlay J, Soerjomataram I, Dikshit R, Eser S, Mathers C, Rebelo M, Parkin DM, Forman D and Bray F: Cancer incidence and mortality worldwide: Sources, methods and major patterns in GLOBOCAN 2012. Int J Can 136: E359-E386, 2014.

2. Van Cutsem E, Nordlinger B, Cervantes A; ESMO Guidelines Working Group: Advanced colorectal cancer: ESMO Clinical Practice Guidelines for treatment. Ann Oncol 21 (Suppl 5): v93-v97, 2010.

3. Saltz LB, Clarke S, Díaz-Rubio E, Scheithauer W, Figer A, Wong R, Koski S, Lichinitser M, Yang TS, Rivera F, et al: Bevacizumab in combination with oxaliplatin-based chemotherapy as first-line therapy in metastatic colorectal cancer: A randomized phase III study. J Clin Oncol 26: 2013-2019, 2008.
4. Bennouna J, Sastre J, Arnold D, Österlund P, Greil R, Van Cutsem E, von Moos R, Viéitez JM, Bouché O, Borg C, et al: Continuation of bevacizumab after first progression in metastatic colorectal cancer (ML18147): A randomised phase 3 trial. Lancet Oncol 14: 29-37, 2013

5. Hurwitz HI, Tebbutt NC, Kabbinavar F, Giantonio BJ, Guan ZZ, Mitchell L, Waterkamp D and Tabernero J: Efficacy and safety of bevacizumab in metastatic colorectal cancer: Pooled analysis from seven randomized controlled trials. Oncologist 18: 1004-1012, 2013.

6. Pai SG and Fuloria J: Novel therapeutic agents in the treatment of metastatic colorectal cancer. World J Gastrointest Oncol 8: 99-104, 2016

7. Spiliopoulou P and Arkenau HT: Rationally designed treatment for metastatic colorectal cancer: Current drug development strategies. World J Gastroenterol 20: 10288-10295, 2014.

8. Bhartiya D and Scaria V: Genomic variations in non-coding RNAs: Structure, function and regulation. Genomics 107: 59-68, 2016.

9. Vychytilova-Faltejskova P, Radova L, Sachlova M, Kosarova Z, Slaba K, Fabian P, Grolich T, Prochazka V, Kala Z, Svoboda M, et al: Serum-based microRNA signatures in early diagnosis and prognosis prediction of colon cancer. Carcinogenesis 37: 941-950, 2016.

10. Mishra PJ: MicroRNAs as promising biomarkers in cancer diagnostics. Biomark Res 2: 19, 2014.

11. Cekaite L, Eide PW, Lind GE, Skotheim RI and Lothe RA: MicroRNAs as growth regulators, their function and biomarker status in colorectal cancer. Oncotarget 7: 6476-6505, 2016.

12. Boisen MK, Dehlendorff C, Linnemann D, Nielsen BS, Larsen JS, Osterlind K, Nielsen SE, Tarpgaard LS, Qvortrup C, Pfeiffer P, et al: Tissue microRNAs as predictors of outcome in patients with metastatic colorectal cancer treated with first line capecitabine and oxaliplatin with or without bevacizumab. PLoS One 15: e109430, 2014.

13. Hansen TF, Carlsen AL, Heegaard NH, Sørensen FB and Jakobsen A: Changes in circulating microRNA-126 during treatment with chemotherapy and bevacizumab predicts treatment response in patients with metastatic colorectal cancer. Br J Cancer 112: 624-629, 2015.

14. Eisenhauer EA, Therasse P, Bogaerts J, Schwartz LH, Sargent D, Ford R, Dancey J, Arbuck S, Gwyther S, Mooney M, et al: New response evaluation criteria in solid tumours: Revised RECIST guideline (version 1.1). Eur J Cancer 45: 228-247, 2009.

15. Carvalho BS and Irizarry RA: A framework for oligonucleotide microarray preprocessing. Bioinformatics 26: 2363-2367, 2010.

16. R Development Core Team: R: a language and environment for statistical computing. R Foundation for Statistical Computing. Vienna, Austria. http://www.r-project.org/. Accessed March 31, 2015.

17. Smyth GK: Limma: linear models for microarray data. In: Bioinformatics and Computational Biology Solutions using R and Bioconductor. Gentleman R, Carey V, Dudoit S, Irizarry R and Huber W (eds). Springer, New York, NY, pp397-420, 2005.

18. Livak KJ and Schmittgen TD: Analysis of relative gene expression data using real-time quantitative PCR and the 2(-Delta Delta C(T)) Method. Methods 25: 402-408, 2001.

19. Vandesompele J, De Preter K, Pattyn F, Poppe B, Van Roy N, De Paepe A and Speleman F: Accurate normalization of real-time quantitative RT-PCR data by geometric averaging of multiple internal control genes. Genome Biol 3: RESEARCH0034, 2002.

20. Schee K, Lorenz S, Worren MM, Günther CC, Holden M, Hovig E, Fodstad O, Meza-Zepeda LA and Flatmark K: Deep sequencing the microRNA transcriptome in colorectal cancer. PLoS One 8: e66165, 2013.

21. Segersten U, Spector Y, Goren Y, Tabak S and Malmström PU: The role of microRNA profiling in prognosticating progression in Ta and T1 urinary bladder cancer. Urol Oncol 32: 613-618, 2014.

22. Zhi Y, Xie X, Wang R, Wang B, Gu W, Ling Y, Dong W, Zhi F and Liu Y: Serum level of miR-10-5p as a prognostic biomarker for acute myeloid leukemia. Int J Hematol 102: 296-303, 2015.

23. Benson EA, Skaar TC, Liu Y, Nephew KP and Matei D: Carboplatin with decitabine therapy, in recurrent platinum resistant ovarian cancer, alters circulating mirnas concentrations: A pilot study. PLoS One 10: e0141279, 2015.

24. Gattolliat CH, Uguen A, Pesson M, Trillet K, Simon B, Doucet L, Robaszkiewicz M and Corcos L: MicroRNA and targeted mRNA expression profiling analysis in human colorectal adenomas and adenocarcinomas. Eur J Cancer 51: 409-420, 2015. 
25. Ozcan O, Kara M, Yumrutas O, Bozgeyik E, Bozgeyik I and Celik OI: MTUS1 and its targeting miRNAs in colorectal carcinoma: Significant associations. Tumour Biol 37: 6637-6645, 2015.

26. Xu Y, Huang Z and Liu Y: Reduced miR-125a-5p expression is associated with gastric carcinogenesis through the targeting of E2F3. Mol Med Rep 10: 2601-2608, 2014.

27. Ferracin M, Lupini L, Salamon I, Saccenti E, Zanzi MV, Rocchi A, Da Ros L, Zagatti B, Musa G, Bassi C, et al: Absolute quantification of cell-free microRNAs in cancer patients. Oncotarget 6: 14545-14555, 2015.
28. Muz B, de la Puente P, Azab F and Azab AK: The role of hypoxia in cancer progression, angiogenesis, metastasis, and resistance to therapy. Hypoxia (Auckl) 3: 83-92, 2015.

29. Agrawal R, Pandey P, Jha P, Dwivedi V, Sarkar C and Kulshreshtha R: Hypoxic signature of microRNAs in glioblastoma: Insights from small RNA deep sequencing. BMC Genomics 15: 686, 2014. 